\title{
Cyclin-dependent kinases 4 and 6 inhibitors (CDK4/6i) versus chemotherapy in luminal $B$ early breast cancer: lessons from the CORALLEEN trial
}

\author{
Claudia De Angelis ${ }^{1}$, Michail Ignatiadis ${ }^{1}$, Martine Piccart-Gebhart ${ }^{1,2}$ \\ ${ }^{1}$ Medical Oncology Department, Academic Trials Promoting Team, Institut Jules Bordet, Université Libre de Bruxelles (U.L.B.), Brussels, Belgium; \\ ${ }^{2}$ Institut Jules Bordet, Université Libre de Bruxelles (U.L.B.), Brussels, Belgium \\ Correspondence to: Prof. Martine Piccart-Gebhart. Scientific Director, Institut Jules Bordet, Université Libre de Bruxelles (U.L.B.), Boulevard de \\ Waterloo 121, 1000 Bruxelles, Brussels, Belgium. Email: martine.piccart@bordet.be. \\ Comment on: Prat A, Saura C, Pascual T, et al. Ribociclib plus letrozole versus chemotherapy for postmenopausal women with hormone receptor-positive, \\ HER2-negative, luminal B breast cancer (CORALLEEN): an open-label, multicentre, randomised, phase 2 trial. Lancet Oncol 2020;21:33-43.
}

Submitted Apr 08, 2020. Accepted for publication May 06, 2020.

doi: $10.21037 /$ atm-20-3216

View this article at: http://dx.doi.org/10.21037/atm-20-3216

About $70 \%$ of all invasive breast cancers are hormone receptor (HR)-positive and human epidermal growth factor receptor 2 (HER2)-negative (1). Cyclin-dependent kinases 4 and 6 inhibitors (CDK4/6i) plus endocrine therapy represents nowadays the standard of care for pre- and postmenopausal women with HR-positive/HER2-negative advanced breast cancer both in the first- and secondline setting (2-5). Six studies have reported the efficacy of CDK4/6i in the neoadjuvant setting: PALLET, NeoPalAna, neoMONARCH, N007, CORALLEEN and NeoPAL (6-11). However, only the last two trials randomized patients between neoadjuvant endocrine treatment plus CDK4/6i $v s$. neoadjuvant chemotherapy $(10,11)$. CORALLEEN, the study that is the main focus of this editorial, is a parallel arm, randomized, phase II trial conducted in 21 centers in Spain in which 106 postmenopausal women with HRpositive/HER2-negative, stage I-IIIA, luminal B by PAM50 (Prosigna) breast cancer were enrolled. A total of 52 patients received six cycles of ribociclib $(600 \mathrm{mg}$ daily for 21 days, every 4 weeks) plus letrozole ( $2.5 \mathrm{mg}$ daily), while 54 patients received four cycles of doxorubicin $\left(60 \mathrm{mg} / \mathrm{m}^{2}\right)$ and cyclophosphamide $\left(600 \mathrm{mg} / \mathrm{m}^{2}\right)$ every 3 weeks followed by 12 cycles of weekly paclitaxel $\left(80 \mathrm{mg} / \mathrm{m}^{2}\right)$. A PAM50 risk of relapse (ROR) prognostic score was evaluated for each patient at study entry. PAM50 ROR score was calculated by integrating gene expression data, tumor size and nodal status and three classes were assigned, namely low, intermediate and high ROR. Most patients had T2 tumors, no axillary node involvement, Ki67 expression level $>14 \%$, and a high-ROR disease at baseline (Table 1) (10). NeoPAL is also a non-comparative, phase II study in which 106 patients with HR-positive/HER2-negative, stage IIIII, luminal B or node-positive luminal A PAM50-defined breast cancer were enrolled. A total of 53 patients received palbociclib (125 mg daily, 3 weeks on and 1 week off) plus letrozole $(2.5 \mathrm{mg}$ daily) for 19 weeks, while 53 patients received three cycles of FEC100 (5-fluorouracil $500 \mathrm{mg} / \mathrm{m}^{2}$, epirubicin $100 \mathrm{mg} / \mathrm{m}^{2}$, and cyclophosphamide $500 \mathrm{mg} / \mathrm{m}^{2}$, every 3 weeks) followed by three cycles of docetaxel $\left(100 \mathrm{mg} / \mathrm{m}^{2}\right.$, every 3 weeks). Similarly, to the Spanish study, most patients in NeoPAL had T2, luminal B, Ki67 expression $\geq 14 \%$, and high-ROR breast cancers. However, in the NeoPAL trial patients with luminal A, node-positive disease were also allowed (Table 1) (11). Two strengths of both trials were: (I) a strong chemotherapy regimen in the non CDK4/6i arm; (II) the patient selection through a prognostic gene signature (PAM50), thus obtaining a homogenous study population $(10,11)$. In a cohort of 1,691 patients with available PAM50 results, patients with luminal A subtype had a lower cumulative probability of dying from breast cancer compared to those with luminal B (7.1\% vs. $16.2 \%$ at 10 years) (12).

How did CDK4/6i perform compared to chemotherapy in terms of clinical and radiological response? In the CORALLEEN study, chemotherapy seemed to be superior to $\mathrm{CDK} 4 / 6 \mathrm{i}$ in terms of objective response rate (ORR) 
Table 1 Baseline characteristics in the CORALLEEN and NeoPAL trials

\begin{tabular}{|c|c|c|c|c|}
\hline Characteristics & \multicolumn{2}{|c|}{ CORALLEEN trial } & \multicolumn{2}{|c|}{ NeoPAL trial } \\
\hline \multicolumn{5}{|l|}{ Age, years } \\
\hline Median [range) & $63.0[56.5-70.3]$ & $64.0[58.3-71.8]$ & 65 [49-78] & 62 [48-80] \\
\hline \multicolumn{5}{|c|}{ Tumour size, n (\%) } \\
\hline $\mathrm{T} 2$ & $40[77]$ & $43[80]$ & $38(73.1)$ & $36(72.0)$ \\
\hline T3 & $9[17]$ & $8[15]$ & $4(7.7)$ & $1(2.0)$ \\
\hline \multicolumn{5}{|c|}{ Lymph node status, n (\%) } \\
\hline NO & $31[60]$ & $31[57]$ & $23(43.4)$ & $25(47.2)$ \\
\hline \multicolumn{5}{|c|}{ Tumour stage, n (\%) } \\
\hline I & $2[4]$ & $2[4]$ & - & - \\
\hline II & $43[83]$ & $45[83]$ & $49(92.4)$ & $52(98.1)$ \\
\hline III & $7[14]$ & $7[13]$ & $4(7.6)$ & $1(1.9)$ \\
\hline \multicolumn{5}{|c|}{ Histological type, n (\%) } \\
\hline Invasive NOS & $41[79]$ & 48 [89] & $44(83.0)$ & $41(77.4)$ \\
\hline Lobular & 10 [19] & $6[11]$ & $7(13.2)$ & $10(18.8)$ \\
\hline Other & $1[2]$ & $0[0]$ & $2(3.8)$ & $2(3.8)$ \\
\hline Luminal A & - & - & $6(11.3)$ & $6(11.3)$ \\
\hline Luminal B & $52[100]$ & $54[100]$ & $47(88.7)$ & $47(88.7)$ \\
\hline \multicolumn{5}{|c|}{ Ki67 expression, n (\%) } \\
\hline$\leq 14 \%$ & $3[6]$ & $2[4]$ & $15(28.3)^{\ddagger}$ & $18(34.0)^{\ddagger}$ \\
\hline$>14 \%$ & 49 [94] & $51[94]^{\dagger}$ & $38(71.7)^{\S}$ & $35(66.0)^{\S}$ \\
\hline \multicolumn{5}{|c|}{ ROR risk class, n (\%) } \\
\hline Intermediate & 8 [15] & $6[11]$ & 7 (13.2) & $9(17.0)$ \\
\hline High & $44[85]$ & 48 [89] & $46(86.8)$ & 44 (83.0) \\
\hline
\end{tabular}

${ }^{\dagger}$, Ki67 value is missing for 1 patient in chemotherapy arm in the CORALLEEN trial; ${ }^{\ddagger}$, patients with Ki67 <14\% in the NeoPAL trial; $\S$, patients with $\mathrm{Ki} 67 \geq 14 \%$ in the NeoPAL trial. NOS, not otherwise specified. 
Table 2 Efficacy parameters in the CORALLEEN and NeoPAL trials

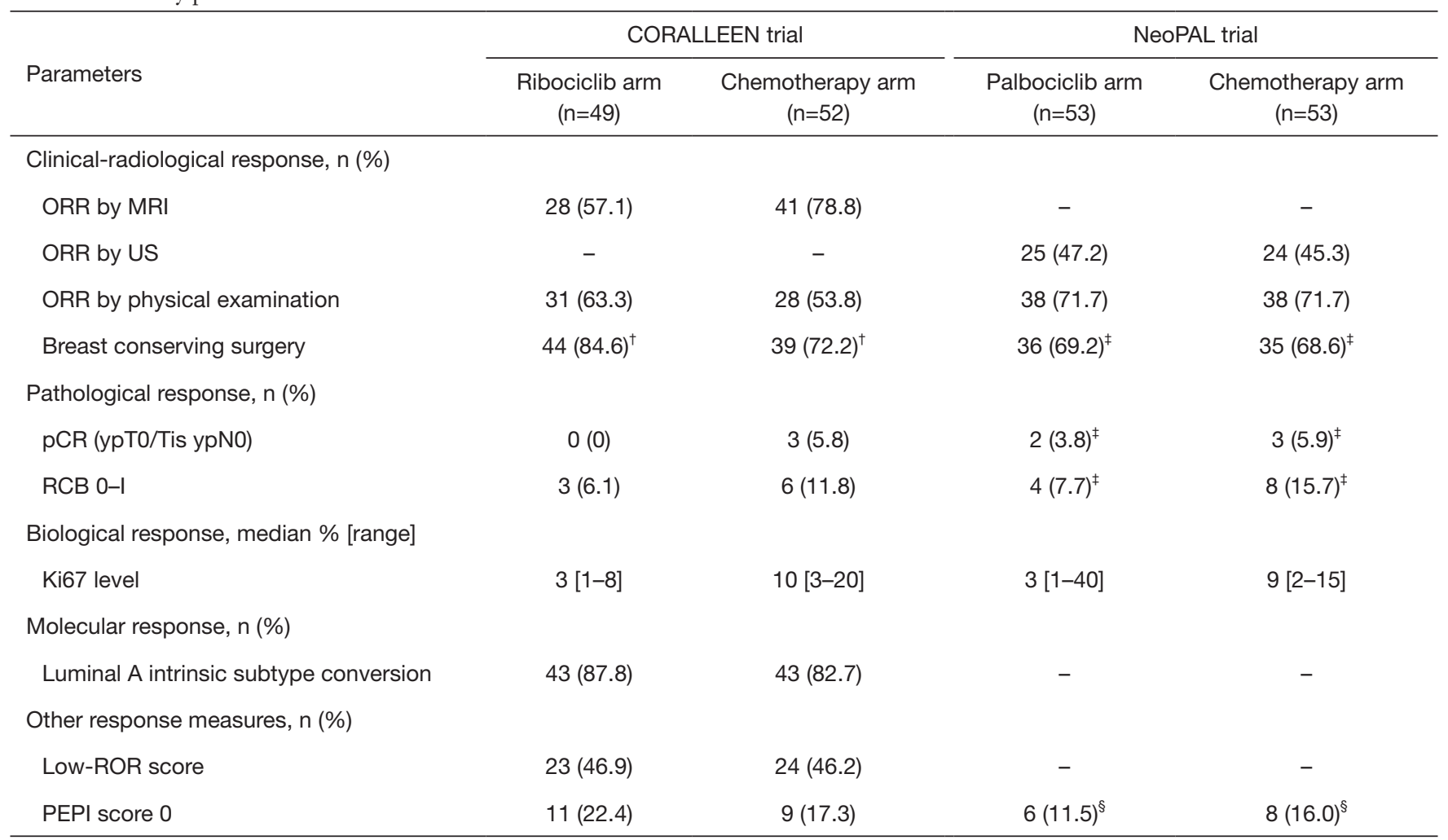

${ }^{\dagger}$, in the CORALLEEN trial, 44 out of 52 evaluable patients in ribociclib arm and 39 out of 54 evaluable patients in chemotherapy arm received breast conserving surgery. ${ }^{\ddagger}$, in the NeoPAL trial, patients evaluable for breast conserving surgery, pCR and RCB were 52 and 51 in palbociclib arm and chemotherapy arm, respectively; PCR and RCB results were reported as per central review. ${ }^{\S}$, PEPI score for relapse-free survival was tested in 52 patients in palbociclib arm and 50 patients in chemotherapy arm; 9 out of 51 (17.6\%) evaluable patients in palbociclib arm and 4 out of 50 (8.0\%) evaluable patients in chemotherapy arm reported PEPI score 0 for breast cancer-specific survival. ORR, objective response rate; US, ultrasound; MRI, magnetic resonance imaging; pCR, pathologic complete response; Tis, tumour in situ; RCB, residual cancer burden; ROR, risk of relapse; PEPI, preoperative endocrine prognostic index.

assessed by magnetic resonance imaging (MRI). However, more patients in ribociclib arm received breast conserving surgery compared to chemotherapy arm. No differences between the two arms were suggested when ORR was evaluated by physical examination (Table 2) (10). NeoPAL also showed a similar ORR between the two treatment arms whether physical examination or ultrasound were used. Indeed, in this trial the same percentage of patients underwent breast conserving surgery in the two arms (Table 2) (11). Pathologic complete response (pCR) and residual cancer burden (RCB) of $0-\mathrm{I}$ were low in both trials, with patients treated with chemotherapy appearing more likely to achieve pCR and RCB 0-I compared to the ones treated with CDK4/6i (Table 2) $(10,11)$. Of note, pCR has been associated with improved eventfree survival [hazard ratio $0.27,95 \%$ confidence interval
(CI): 0.14-0.50] in high proliferative, HR-positive/ HER2-negative breast cancer (13). Moving to biological response, both trials showed greater reduction in Ki67 expression levels with CDK4/6i than with chemotherapy (Table 2) $(10,11)$. Of note, this decrease in Ki67 could be underestimated due to Ki67 rebound occurring when CDK4/6i are suspended some days before surgery, as the NeoPalAna study has previously shown (7). This issue is mainly applicable to the CORALLEEN trial where 13.1 [standard deviation (SD) 14.6] was the mean number of days between the last dose of ribociclib and surgery (10). Conversely, in NeoPAL, patients should have been operated 24 hours after the last dose of palbociclib (11). However, in this trial the median time between the last dose of palbociclib and surgery was not reported $(10,11)$. The conversion to luminal A subtype 
assessed by PAM50 at surgery was evaluated only in the CORALLEEN trial. A numerically higher percentage of conversion to luminal A subtype was observed in the ribociclib arm compared to chemotherapy arm (Table 2) (10). Thus, CDK4/6i could be at least as effective as chemotherapy in terms of Ki67 reduction and conversion to luminal A subtype. This was also the case when efficacy was assessed by combining clinicopathological and molecular variables. In the CORALLEEN study, similar rates of low-ROR score at surgery were found in the ribociclib and chemotherapy arms (10). ROR score was evaluated exclusively at baseline in the NeoPAL trial (Table 2) (11). Despite some numerical differences, similar percentages of patients with chemotherapy or CDK4/6i in both trials achieved preoperative endocrine prognostic index (PEPI) score 0 (Table 2) $(10,11)$, known to be associated to more favorable relapse-free survival (14).

Results reported in the other four neoadjuvant trials are in line with those seen in the CDK4/6i arm of CORALLEEN and NeoPAL (6-9). In the NeoPalAna trial, patients received 4 weeks of anastrozole followed by 4 cycles of palbociclib plus anastrozole before surgery. In line with NeoPAL trial, $80.5 \%$ and $40.7 \%$ of patients reached ORR by physical examination and by ultrasound respectively, and no pCR was recorded. At the biological level, complete cell cycle arrest (CCCA, defined as Ki67 $\leq 2.7 \%$ ) rate was significantly higher at cycle (C) 1 day (D) 15 with palbociclib plus anastrozole than that at C1D1 with anastrozole monotherapy ( $87 \%$ vs. $26 \% ; \mathrm{P}<0.001)$, both in luminal $\mathrm{A}(\mathrm{P}=0.008)$ and luminal $\mathrm{B}(\mathrm{P}=0.02)$ tumors (PAM50-defined) at baseline. Furthermore, PAM50 proliferation score (using a 11-gene signature) was significantly reduced from C1D1 to C1D15 $(\mathrm{P}<0.0001)$ and correlated with Ki67 drop at all timepoints (7). Also, in the PALLET study, there was no evidence that the addition of palbociclib to letrozole increased ORR measured by ultrasound $(54.4 \%$ vs. $49.5 \% ; \mathrm{P}=0.20)$ or $\mathrm{pCR}$ rates $(1.1 \%$ vs. $3.3 \% ; \mathrm{P}=0.43)$. Moreover, no difference in the proportion of patients that have undergone breast conserving surgery was seen $(60.8 \%$ in palbociclib arm vs. $59.2 \%$ in letrozole arm). Conversely, median log-fold change in Ki67 (-4.1 vs. $-2.2 ; \mathrm{P}<0.001$ ) and poly(ADP-ribose) polymerase (apoptosis, -0.80 vs. $-0.42 ; \mathrm{P}<0.001)$ was greater with palbociclib plus letrozole than letrozole alone, and there was a significant difference in CCCA (90.4\% vs. $58.5 \%$; $\mathrm{P}<0.001)$, too (6). In the neoMONARCH study, Ki67 at week 2 decreased by a geometric mean change of $-93 \%$ in the abemaciclib plus anastrozole arm, $-91 \%$ in the abemaciclib arm, and
$-63 \%$ in the anastrozole arm $(\mathrm{P}<0.001)$. Moreover, patients treated with abemaciclib \pm anastrozole reported a significant higher CCCA compared to anastrozole alone after 2 weeks $(\mathrm{P}<0.001)$. Furthermore, abemaciclib \pm anastrozole led to a significant decrease of cell cycle-associated genes (FOXM1, RRM2, CCNE1, MKI67, TOPO2A) at 2 weeks $(\mathrm{P}<0.05)(8)$. Finally, in the small N007 study of 20 patients that received palbociclib and endocrine treatment, a clinical ORR of $85 \%$ and ORR by ultrasound of $70 \%$ were observed, and only one pCR was recorded. The mean value of Ki67 (P=0.044) and proliferation-associated genes (BIRC5, UBE2C and DHCR 7; $\mathrm{P}<0.001)$, significantly decreased from baseline to surgery. A significant reduction in mean EndoPredict (EP) score occurred from baseline to surgery (6.87 vs. 5.25; $\mathrm{P}<0.0001)$ as well. Only 1 patient at surgery had PEPI 0 score, and 9 patients reached low EP clin score (9).

How do CDK4/6i compare to chemotherapy in the metastatic setting? Only 2 reported trials have directly addressed this question. The first one, is a phase III trial (PEARL) that enrolled 601 postmenopausal patients with HR-positive/HER2-negative metastatic breast cancer resistant to previous non-steroidal aromatase inhibitors (AIs), and allocated them to receive palbociclib plus exemestane (cohort 1)/fulvestrant [cohort 2, added after publication of data about ESR1 mutations as mechanism of resistance to AIs (15)] or capecitabine alone. Palbociclib plus fulvestrant did not show a different median progressionfree survival (PFS) compared to capecitabine alone [7.5 vs. 10 months (m); adjusted hazard ratio $1.09,95 \% \mathrm{CI}$ : 0.83-1.44; $\mathrm{P}=0.537$ ]. Similarly, there was no improvement in median PFS in ESR1 wild-type patients treated with palbociclib plus fulvestrant/exemestane compared to capecitabine alone $(8.0$ vs. $10.6 \mathrm{~m}$; adjusted hazard ratio $1.08,95 \%$ CI: $0.85-1.36 ; \mathrm{P}=0.526)$. In terms of ORR, palbociclib plus fulvestrant was not superior to capecitabine (26.7\% vs. 33.3\%) (16). The second study (Young PEARL), was a phase II trial, in which 184 premenopausal women with HR-positive/HER2-negative metastatic breast cancer were randomized to receive palbociclib, exemestane, and leuprolide or capecitabine. All patients were previously treated with tamoxifen. Contrary to the PEARL study, the palbociclib arm showed a significant increase in median PFS compared to the chemotherapy arm (20.1 vs. $14.4 \mathrm{~m}$; hazard ratio $0.659,95 \%$ CI: $0.437-0.994 ; \mathrm{P}=0.0235)$. No difference in ORR was detected $(37 \%$ vs. $34 \%$ in the palbociclib and capecitabine arms, respectively; $\mathrm{P}=0.781$ ) (17). In a network meta-analysis, no chemotherapy was significantly better than CDK4/6i and endocrine treatment in terms of PFS, 
while CDK4/6i plus endocrine treatment was better than endocrine treatment alone (18). Thus, given the favorable toxicity profile of CDK4/6i over chemotherapy, this class of agents is the preferred option for $1^{\text {st }}$ line treatment according to different guidelines. However, the metastatic setting is completely different from the early setting. In the early setting cure is the goal and thus strong data from a phase 3 trial are needed in order to demonstrate a noninferiority of a CDK4/6i, chemotherapy-free regimen. We are not aware of any such trial ongoing in the general population. There is one trial in the elderly population, the European Organization for the Research and Treatment of Cancer (EORTC) APPALACHES trial that evaluates a chemotherapy-free, CDK4/6i and endocrine adjuvant treatment in elderly patients that will be otherwise candidates for adjuvant chemotherapy (NCT03609047).

Thus, we think that the CORALLEEN study could be most interesting in terms of identifying the patients that are resistant to CDK4/6i. Future translational research aiming to understand the mechanisms of primary treatment resistance in these patients is warranted. Apart from Ki67 and PAM50, no other biomarkers have been evaluated in the CORALLEEN and NeoPAL trials, so far. PIK3CA mutation status has not been associated with sensitivity to CDK4/6i. Indeed, in NeoPalAna and neoMONARCH studies Ki67 suppression and CCCA at C1D15 occurred regardless of PIK3CA mutation $(7,8)$. In the PALOMA-3 trial, the addition of palbociclib to fulvestrant improved PFS regardless of PIK3CA mutation status (19). In the NeoPalAna study, significantly elevated mRNA expression levels of CCND3, CCNE1, and CDKN2D genes at C1D15 were found in the resistant group (7). Also, in the neoMONARCH study numerically high mRNA expression levels of $C C N E 1$ and $E 2 F 1$ at week 2 were found in resistant patients (8). These findings are supported by the PALOMA-3 trial results where high median CCNE1 mRNA expression correlated with worse PFS (false discovery rateadjusted $\mathrm{P}=0.0238$ ) (20). Moreover, low expression of $R B 1$ mRNA was associated with resistance to abemaciclib monotherapy in the neoMONARCH trial (8). This is in line with previous results showing that inactivating mutations in the $R B 1$ gene might be associated with resistance to CDK4/6i (21). Genomic alterations of the fibroblast growth factor receptor (FGFR) or Hippo pathways have recently emerged as mechanisms of resistance to CDK4/6i in the metastatic setting $(22,23)$, but they have not yet been explored in the early setting. Interesting data about immunomodulation mediated by CDK4/6i in the early setting emerged from the neoMONARCH trial where the combination of abemaciclib plus anastrozole enhanced immune response genes related to INF $\gamma$ production and PD-1 signaling, without increasing stromal tumorinfiltrating lymphocytes (TIL) levels (8). This is consistent with preclinical studies suggesting that CDK4/6i exposure might enhance the anti-tumor immune response (24). Results from ongoing, chemotherapy-free studies evaluating the combination of CDK4/6i and immunotherapy in the neoadjuvant setting (NCT03573648, NCT04075604, NCT04088032) are eagerly awaited.

In conclusion, although low-ROR score was observed at surgery in almost half of the patients treated with letrozole and ribociclib in the CORALLEEN trial, the prognostic significance of this low-ROR score at surgery has not been established as acknowledged by the authors. Thus, no statement can be made for these patients concerning their need or not for adjuvant chemotherapy. The role of adjuvant CDK4/6i in the early setting will be clarified from the large ongoing phase 3 adjuvant trials (NCT01864746, NCT02513394, NCT03155997, NCT03701334). The different neoadjuvant studies might serve as translational research platforms to help understand resistance to CDK4/6i but ultimately the results from these studies will need to be validated in the adjuvant studies. As of today, 806 patients have been enrolled in the six published neoadjuvant CDK4/6i trials, and $>2,000$ patients are being enrolled in additional neoadjuvant CDK4/6i trials (25). A collaborative effort using the samples from patients treated with CDK4/6i in the above neoadjuvant trials might provide important insights into the mechanisms of resistance to this class of compounds and help oncologists with an improved selection of women who may be spared chemotherapy.

\section{Acknowledgments}

Funding: None.

\section{Footnote}

Provenance and Peer Review: This article was commissioned by the editorial office, Annals of Translational Medicine. The article did not undergo external peer review.

Conflicts of Interest: All authors have completed the ICMJE uniform disclosure form (available at http://dx.doi. org/10.21037/atm-20-3216). MI declares: Consultant or advisory role: Celgene, Novartis, Pfizer, Seattle 
Genetics, Tesaro; Speaker honoraria: Novartis; Travel grants: Pfizer, Amgen; Research grants to my Institute: Roche, Menarini Silicon Biosystems, Janssen Diagnostics, Pfizer; MPG declares: Board member (scientific board): Oncolytics; Consultant (honoraria): AstraZeneca, CamelIDS, Crescendo Biologics, Debiopharm, G1 Therapeutics, Genentech, Huya, Immunomedics, Lilly, Menarini, MSD, Novartis, Odonate, Periphagen, Pfizer, Roche, Seattle Genetics; Research grants to my Institute: AstraZeneca, Lilly, MSD, Novartis, Pfizer, Radius, Roche-Genentech, Servier, Synthon. The other author has no other conflicts of interest to declare.

Ethical Statement: The authors are accountable for all aspects of the work in ensuring that questions related to the accuracy or integrity of any part of the work are appropriately investigated and resolved.

Open Access Statement: This is an Open Access article distributed in accordance with the Creative Commons Attribution-NonCommercial-NoDerivs 4.0 International License (CC BY-NC-ND 4.0), which permits the noncommercial replication and distribution of the article with the strict proviso that no changes or edits are made and the original work is properly cited (including links to both the formal publication through the relevant DOI and the license). See: https://creativecommons.org/licenses/by-nc-nd/4.0/.

\section{References}

1. Parker JS, Mullins M, Cheang MCU, et al. Supervised risk predictor of breast cancer based on intrinsic subtypes. J Clin Oncol 2009;27:1160-7.

2. Im SA, Lu YS, Bardia A, et al. Overall Survival with Ribociclib plus Endocrine Therapy in Breast Cancer. N Engl J Med 2019;381:307-16.

3. Slamon DJ, Neven P, Chia S, et al. Overall Survival with Ribociclib plus Fulvestrant in Advanced Breast Cancer. N Engl J Med 2020;382:514-24.

4. Sledge GW Jr, Toi M, Neven P, et al. The Effect of Abemaciclib Plus Fulvestrant on Overall Survival in Hormone Receptor-Positive, ERBB2-Negative Breast Cancer That Progressed on Endocrine TherapyMONARCH 2: A Randomized Clinical Trial. JAMA Oncol 2019;6:116-24.

5. Turner NC, Ro J, André F, et al. Palbociclib in HormoneReceptor-Positive Advanced Breast Cancer. N Engl J Med 2015;373:209-19.
6. Johnston S, Puhalla S, Wheatley D, et al. Randomized Phase II Study Evaluating Palbociclib in Addition to Letrozole as Neoadjuvant Therapy in Estrogen ReceptorPositive Early Breast Cancer: PALLET Trial. J Clin Oncol 2019;37:178-89.

7. Ma CX, Gao F, Luo J, et al. NeoPalAna: Neoadjuvant Palbociclib, a Cyclin-Dependent Kinase 4/6 Inhibitor, and Anastrozole for Clinical Stage 2 or 3 Estrogen ReceptorPositive Breast Cancer. Clin Cancer Res 2017;23:4055-65.

8. Hurvitz SA, Martin M, Press MF, et al. Potent Cell-Cycle Inhibition and Upregulation of Immune Response with Abemaciclib and Anastrozole in neoMONARCH, Phase II Neoadjuvant Study in HR+/HER2- Breast Cancer. Clin Cancer Res 2020;26:566-80.

9. Chow LWC, Morita S, Chow CYC, et al. Neoadjuvant palbociclib on ER+ breast cancer (N007): clinical response and EndoPredict's value. Endocr Relat Cancer 2018;25:123-30.

10. Prat A, Saura C, Pascual T, et al. Ribociclib plus letrozole versus chemotherapy for postmenopausal women with hormone receptor-positive, HER2-negative, luminal B breast cancer (CORALLEEN): an open-label, multicentre, randomised, phase 2 trial. Lancet Oncol 2020;21:33-43.

11. Cottu P, D'Hondt V, Dureau S, et al. Letrozole and palbociclib versus chemotherapy as neoadjuvant therapy of high-risk luminal breast cancer. Ann Oncol 2018;29:2334-40.

12. Caan BJ, Sweeney C, Habel LA, et al. Intrinsic subtypes from the PAM50 gene expression assay in a populationbased breast cancer survivor cohort: prognostication of short- and long-term outcomes. Cancer Epidemiol Biomarkers Prev 2014;23:725-34.

13. Cortazar P, Zhang L, Untch M, et al. Pathological complete response and long-term clinical benefit in breast cancer: the CTNeoBC pooled analysis. Lancet 2014;384:164-72.

14. Ellis MJ, Tao Y, Luo J, et al. Outcome prediction for estrogen receptor-positive breast cancer based on postneoadjuvant endocrine therapy tumor characteristics. J Natl Cancer Inst 2008;100:1380-8.

15. Turner NC, Jiang Y, O'Leary B, et al. Efficacy of palbociclib plus fulvestrant $(\mathrm{P}+\mathrm{F})$ in patients (pts) with metastatic breast cancer (MBC) and ESR1 mutations (mus) in circulating tumor DNA (ctDNA). J Clin Oncol 2016;34:abstr 512.

16. Martín M, Zielinski C, Ruíz-Borrego M, et al. Results from PEARL study (GEICAM/2013-02_CECOG/ BC.1.3.006): A phase 3 trial of Palbociclib (PAL) in 
combination with endocrine therapy (ET) versus Capecitabine (CAPE) in hormonal receptor (HR)positive/human epidermal growth factor receptor (HER) 2-negative metastatic breast cancer (MBC) patients (pts) whose disease progressed on aromatase inhibitors (AIs). Cancer Res 2020;80:abstr GS2-07.

17. Park YH, Kim TY, Kim GM, et al. Palbociclib plus exemestane with gonadotropin-releasing hormone agonist versus capecitabine in premenopausal women with hormone receptor-positive, HER2-negative metastatic breast cancer (KCSG-BR15-10): a multicentre, open-label, randomised, phase 2 trial. Lancet Oncol 2019;20:1750-9.

18. Giuliano M, Schettini F, Rognoni C, et al. Endocrine treatment versus chemotherapy in postmenopausal women with hormone receptor-positive, HER2-negative, metastatic breast cancer: a systematic review and network meta-analysis. Lancet Oncol 2019;20:1360-9.

19. Cristofanilli M, Turner NC, Bondarenko I, et al. Fulvestrant plus palbociclib versus fulvestrant plus placebo for treatment of hormone-receptor-positive, HER2negative metastatic breast cancer that progressed on previous endocrine therapy (PALOMA-3): final analysis

Cite this article as: De Angelis C, Ignatiadis M, PiccartGebhart M. Cyclin-dependent kinases 4 and 6 inhibitors (CDK4/6i) versus chemotherapy in luminal B early breast cancer: lessons from the CORALLEEN trial. Ann Transl Med 2020;8(21):1338. doi: 10.21037/atm-20-3216 of the multicentre, double-blind, phase 3 randomised controlled trial. Lancet Oncol 2016;17:425-39.

20. Turner NC, Liu Y, Zhu Z, et al. Cyclin E1 Expression and Palbociclib Efficacy in Previously Treated Hormone Receptor-Positive Metastatic Breast Cancer. J Clin Oncol 2019;37:1169-78.

21. Bertucci F, Ng CKY, Patsouris A, et al. Genomic characterization of metastatic breast cancers. Nature 2019;569:560-4. Erratum in: Nature. 2019 Aug;572(7767):E7

22. Formisano L, Lu Y, Servetto A, et al. Aberrant FGFR signaling mediates resistance to CDK4/6 inhibitors in ER+ breast cancer. Nat Commun 2019;10:1373.

23. Li Z, Razavi P, Li Q, et al. Loss of the FAT1 Tumor Suppressor Promotes Resistance to CDK4/6 Inhibitors via the Hippo Pathway. Cancer Cell 2018;34:893-905.e8.

24. Goel S, DeCristo MJ, Watt AC, et al. CDK4/6 inhibition triggers anti-tumour immunity. Nature 2017;548:471-5.

25. Brandão $M$, Ignatiadis $M$. CDK4/6 inhibitors as neoadjuvant treatment in breast cancer-what can we learn? Ann Oncol 2018;29:2274-8. 